\title{
Spotlight on brimonidine topical gel $0.33 \%$ for facial erythema of rosacea: safety, efficacy, and patient acceptability
}

This article was published in the following Dove Press journal:

Patient Preference and Adherence

6 July 2017

Number of times this article has been viewed

\author{
Michael S Anderson' \\ Anish Nadkarni' \\ Leah A Cardwell' \\ Hossein Alinia' \\ Steven R Feldman ${ }^{1-3}$ \\ 'Department of Dermatology, \\ Center for Dermatology Research, \\ ${ }^{2}$ Department of Pathology, \\ ${ }^{3}$ Department of Public Health \\ Sciences, Wake Forest School of \\ Medicine, Winston-Salem, NC, USA
}

\begin{abstract}
Background: Brimonidine tartrate is a highly selective alpha 2 agonist that induces direct vasoconstriction of small arteries and veins, thereby reducing vasodilation and edema.

Objective: To review the current literature regarding the safety, efficacy, and patient acceptability of brimonidine $0.33 \%$ gel.

Methods: A PubMed search was performed using the terms brimonidine $0.33 \%$ gel, rosacea, safety, efficacy, and acceptability. Peer-reviewed clinical trials and case reports from 2012 to 2016 were screened for inclusion of safety, efficacy, and/or patient acceptability data.

Results: Brimonidine topical gel $0.33 \%$ is associated with mild, transient skin-related adverse reactions. Efficacy may be achieved within 30 minutes of administration with maximal reductions in erythema 3-6 hours after administration. Patient satisfaction with use of brimonidine topical gel is superior to vehicle gel for facial appearance, treatment effect, facial redness, and daily control of facial redness.
\end{abstract}

Limitations: Studies were typically limited to 1-year follow-up. Only one study has examined the use of brimonidine topical gel in combination with other rosacea and acne medications.

Discussion: Brimonidine topical gel $0.33 \%$ is a safe, effective, and patient-accepted treatment for facial erythema of rosacea.

Keywords: patient satisfaction, adverse reactions, side effects

\section{Introduction}

Rosacea is a chronic inflammatory skin disorder categorized into four types. These include erythematotelangiectatic rosacea (ETR), papulopustular rosacea (PPR), phymatous rosacea (PHY), and ocular rosacea (OR). ETR is characterized by flushing, erythema, and telangiectasias of the central face. PPR features papules and pustules of the central face. Patients with PHY have skin thickening and sebaceous gland hypertrophy leading to skin surface irregularity and disfigurement. OR symptoms include conjunctival hyperemia, anterior blepharitis, and keratitis. ETR is the most prevalent subtype, followed by PPR, OR, and PHY. ${ }^{1}$ However, patients may have features of multiple subtypes of rosacea, complicating treatment regimens. ${ }^{2-4}$

Rosacea most commonly affects fair-skinned women aged $>30$ years. ${ }^{5,6}$ Ninety-six percent of patients with PPR are skin types I-II. ${ }^{7}$ Aggressive treatment is important as rosacea symptoms are frequently associated with lower self-esteem and fear of social stigma. ${ }^{8}$ The pathogenesis of rosacea is not completely understood, but innate immune dysfunction, inflammation, presence of microorganisms, UV damage, and 
neurovascular abnormalities in conjunction with various triggers may play a role in condition manifestation. ${ }^{7}$

Activation of the innate immune system in a susceptible individual may produce cathelicidin peptides that can induce vasodilation and inflammation. ${ }^{9}$ Levels of cathelicidin and kallikrein 5 , a processing enzyme of cathelicidin, are elevated in the skin of patients with rosacea. ${ }^{10}$ Demodex folliculorum mites are present in greater concentrations in the sebaceous follicles of patients with rosacea. ${ }^{11}$ Bacillus oleronius, a bacterium that may colonize Demodex mites, stimulates an inflammatory response at higher rates in patients with rosacea. ${ }^{12}$ Given the distribution of rosacea in sun-exposed areas, ultraviolet radiation may play a role in rosacea pathogenesis. UVB radiation stimulates vascular endothelial growth factor and fibroblast growth factor from keratinocytes in mice. ${ }^{13-15}$ Reactive oxygen species produced by UV damage may also stimulate the innate immune system leading to the symptoms of rosacea. ${ }^{9}$ The transient receptor potential vanilloid (TRPV) ion channel is overexpressed in the sensory nerve endings in patients with rosacea. ${ }^{16-18} \mathrm{TRPV}$, a vasodilator, is activated in response to common triggers of rosacea such as heat, spicy food, and alcohol. It causes vasoactive peptides to be released, leading to vasodilation and flushing. ${ }^{19,20}$

Treatment options for rosacea include topicals such as metronidazole, azelaic acid, ivermectin, brimonidine, oxymetazoline, and sulfacetamides. For PPR, oral metronidazole, doxycycline, or isotretinoin may be employed. Brimonidine and oxymetazoline are approved by the Food and Drug Administration (FDA) for the treatment of the facial erythema associated with rosacea.

Brimonidine is a highly selective alpha 2 agonist that binds alpha 2 receptors on the vasculature inducing direct vasoconstriction of both small arteries and veins, thereby reducing vasodilation and edema. ${ }^{21}$ We aim to summarize the safety, efficacy, and patient acceptability of brimonidine topical gel in the treatment of facial erythema associated with rosacea.

\section{Methods}

A PubMed search was performed using the terms brimonidine $0.33 \%$ gel, brimonidine and rosacea, brimonidine $0.33 \%$ gel and safety, brimonidine safety and rosacea, brimonidine $0.33 \%$ gel and efficacy, brimonidine efficacy and rosacea, and brimonidine acceptability and rosacea. Ten peer-reviewed clinical trials and case reports from 2012 to 2016 were screened for inclusion of safety, efficacy, and/or patient acceptability of brimonidine topical gel. Full text was reviewed; all 10 articles were included as they contained pertinent research data or clinical reports. Review articles were excluded.

\section{Results}

The safety of topical brimonidine tartrate gel for moderateto-severe facial erythema of rosacea was examined in two related Phase II studies (Table 1). ${ }^{22}$ The rates of adverse events were similarly low, ranging from $6 \%$ to $14 \%$, between three different concentrations of brimonidine tartrate $(0.5 \%$, $0.18 \%, 0.07 \%$ ) and vehicle gel after one-time application. When extended to once-daily application for 4 weeks with a 4-week follow-up period, adverse event rates ranged from $11 \%$ to $19 \%$ and were similar across all brimonidine tartrate concentrations. Adverse events were mild, transient, and limited to the skin. Irritation, flushing, worsened erythema, burning sensation, and pruritus were the most commonly reported side effects. Phase III studies of brimonidine tartrate $0.5 \%$ (equivalent to brimonidine topical gel $0.33 \%$ ) noted adverse event rates ranging from $29.5 \%$ to $33.8 \%$ after 4 weeks of once-daily use. The rate of adverse reactions was slightly higher for brimonidine tartrate $0.5 \%$ than for vehicle gel, $29.5 \%$ vs $25.2 \%$ in the one-time treatment group and $33.8 \%$ vs $24.1 \%$ in the 4 -week treatment group..$^{23}$ In a 1 -year open-label study of brimonidine tartrate $0.5 \%$ applied once daily, adverse events were highest during 1-3 months of daily use with a rate of $41.9 \%$. Rates decreased with longer duration of treatment; the adverse event rate was $19.5 \%$ at months 10 through 12 of treatment. Concomitant use of medications to address inflammatory lesions did not increase adverse reactions..$^{24}$ Since its commercial availability in 2013, multiple case reports have documented contact dermatitis and rebound erythema worse than baseline after initial improvement with brimonidine use..$^{25-29}$

Brimonidine topical gel $0.33 \%$ was efficacious compared to vehicle gel for moderate-to-severe persistent facial erythema of rosacea in Phase II, Phase III, and open-label studies (Table 2). There was a dose-dependent improvement in facial erythema with use of brimonidine tartrate as assessed by Clinician's Erythema Assessment (CEA) and Patient Self-Assessment (PSA) tools. ${ }^{23}$ Concentrations of $0.07 \%, 0.18 \%$, and $0.5 \%$ were compared against vehicle gel in both one-time and daily applications for 4 weeks. In the one-time dosing trial, improvement in facial erythema was correlated with increasing brimonidine tartrate concentration. After application of brimonidine tartrate $0.5 \%$ once daily for 4 weeks, there was superior improvement in erythema compared to lower concentrations and vehicle gel. ${ }^{22}$ When used once 


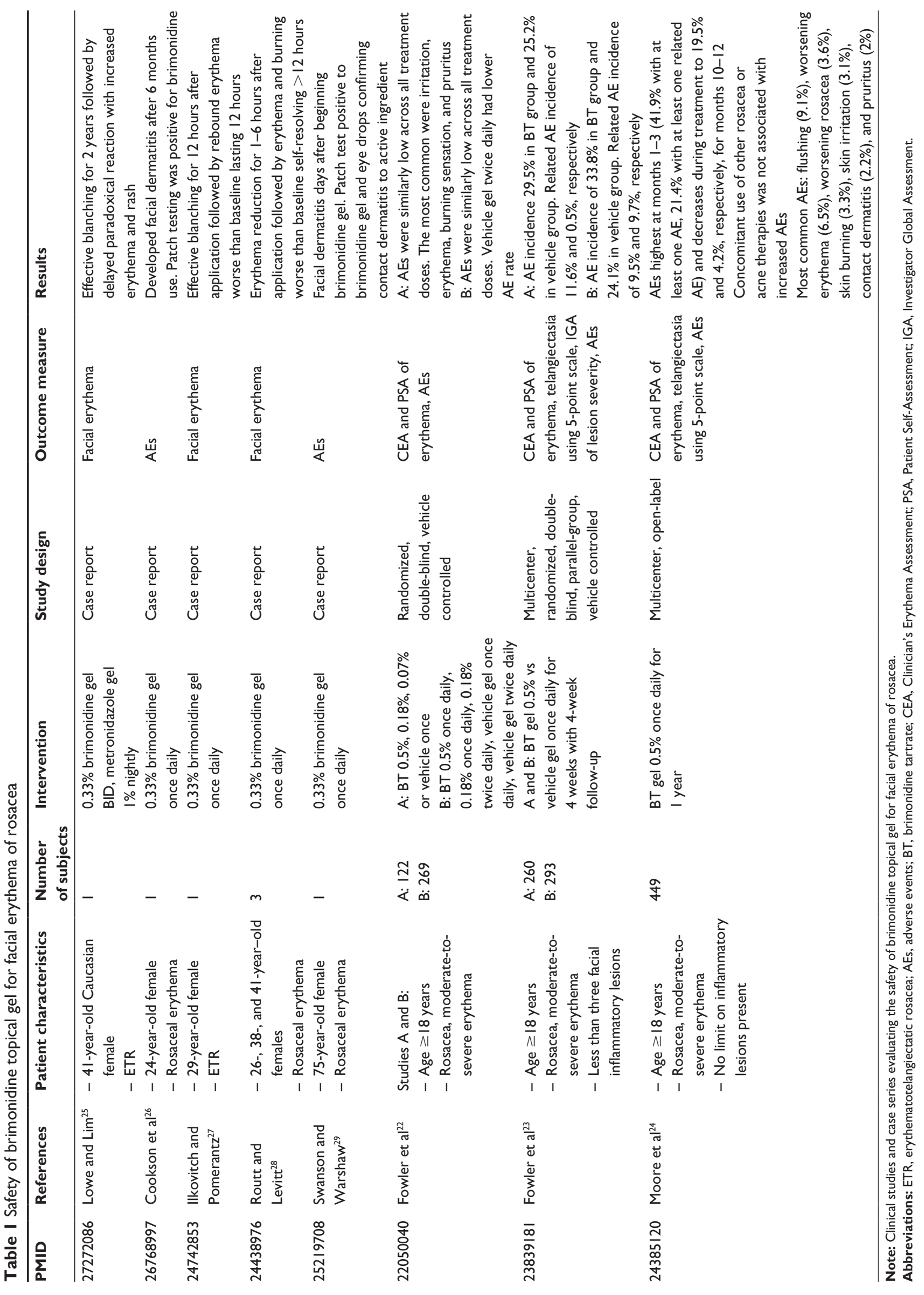




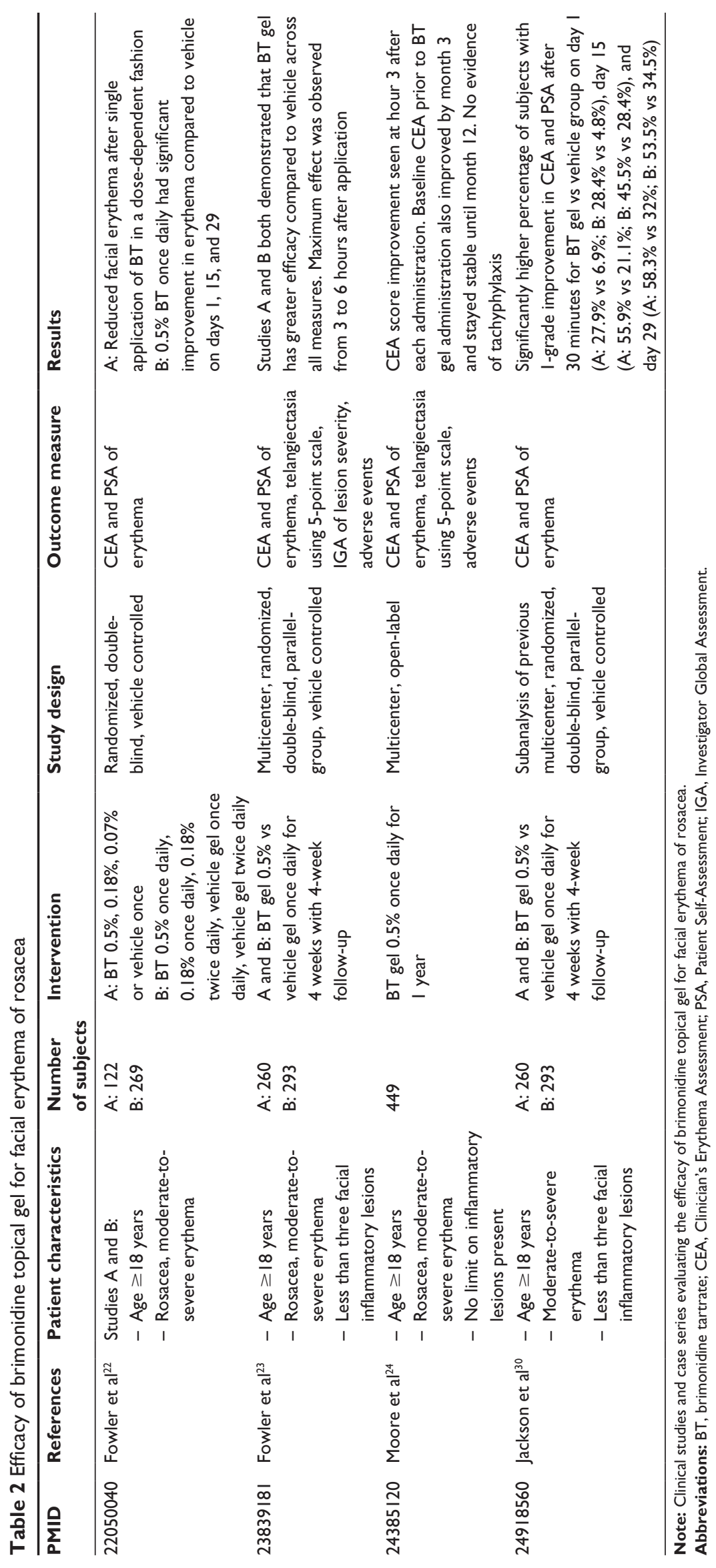


daily for 4 weeks as monotherapy in larger Phase III studies, brimonidine tartrate $0.5 \%$ had greater efficacy than vehicle gel in patients with less than three inflammatory lesions. Maximal effect was observed 3-6 hours after application of brimonidine tartrate $0.5 \%$, with more patients experiencing at least a 2-grade improvement in CEA and PSA compared to vehicle. ${ }^{23}$ Of the subjects treated with brimonidine tartrate $0.5 \%$ gel, $27.9 \%$ achieved a 1 -grade improvement in both CEA and PSA scores 30 minutes after administration compared to vehicle gel ( $6.9 \%$ of subjects) on day 1 . This end point was achieved by $55.9 \%$ of subjects in the brimonidine tartrate $0.5 \%$ group vs $21.1 \%$ of subjects in the vehicle group and $58.3 \%$ of subjects in the brimonidine tartrate $0.5 \%$ group vs $32 \%$ of subjects in the vehicle group on days 15 and 29 , respectively. ${ }^{30} \mathrm{~A} 1$-year open-label study of brimonidine tartrate $0.5 \%$ gel daily evaluated the improvement in erythema based on CEA and PSA with no limit on inflammatory lesions and no restrictions on concomitant acne or rosacea medications. Reductions were continually observed in both CEA and PSA following application of brimonidine tartrate $0.5 \%$ gel throughout the course of the study, with maximal improvement 3 hours after administration. Baseline erythema scores prior to daily administration of brimonidine tartrate appeared to improve over time with continued brimonidine use. Mean PSA score prior to administration of brimonidine tartrate at day 1 of treatment was 3.1 and at month 3 of treatment was 2.3. Mean CEA score prior to administration of brimonidine tartrate decreased from 3.1 on day 1 to 2.4 at month 3 . The baseline erythema improvement was maintained through the end of the 12-month study period. ${ }^{24}$

Patient acceptability of brimonidine topical gel was demonstrated in Phase II, Phase III, and open-label studies (Table 3). Patient acceptability was best quantified by a randomized, double-blind, vehicle-controlled, and parallelgroup study of 92 patients using $1 \mathrm{~g}$ brimonidine topical gel $0.33 \%$ or $1 \mathrm{~g}$ vehicle gel daily for 8 days. The study included patients with self-perceived severe facial erythema and measured patient-reported outcomes using a facial redness questionnaire, satisfaction questionnaire, and diary of facial redness. On comparing the treatment group vs the vehicle group, there was improved satisfaction with facial appearance $(36.9 \%$ vs $21.5 \%)$, treatment effect ( $69.6 \%$ vs $40.4 \%)$, facial redness (67.4\% vs $33.3 \%)$, and daily control of facial redness ( $83 \%$ vs $28.9 \%) .{ }^{31}$

\section{Discussion}

The safety of brimonidine topical gel $0.33 \%$ once-daily dosing is well characterized. Although there is a relatively high rate of adverse reactions, up to $41.9 \%$ with daily use, the reactions are generally mild, transient, and limited to the skin. ${ }^{24}$ The most common reactions are flushing, erythema worse (or at least perceived as worse) than baseline, worsening of rosacea, irritation, and pruritus..$^{22-24}$ These side effects generally present shortly after application or as the effects of the medication are wearing off and resolve within hours without intervention. Side-effect incidence is typically highest from initial use through month 3 of use but decreases with longer duration of daily application. Early studies reported low incidence of contact dermatitis and rebound erythema with topical brimonidine tartrate, but recent case studies suggest that these are potentially significant reactions. Contact dermatitis to either vehicle ingredients or brimonidine occurred in multiple patients. ${ }^{24,26,29}$ Exaggerated rebound erythema $\sim 12$ hours after application of brimonidine topical gel has also been reported. The erythema may be worse than baseline but typically resolves within 6-12 hours. ${ }^{25,27,28}$ These adverse events should be considered when developing treatment regimens. However, given the mild and transient nature of these adverse reactions, a trial of brimonidine topical gel after side-effect counseling may be reasonable.

Brimonidine topical gel $0.33 \%$ is efficacious for moderate-to-severe facial erythema of rosacea, both as monotherapy and in conjunction with other acne and rosacea medications..$^{22-24,30}$ Reduction in erythema is observed 30 minutes after administration, and patients can expect a peak benefit between 3 and 6 hours after administration. Due to the mechanism of action of brimonidine, redness will generally recur within 12 hours after application. Many patients may experience an overall reduction in baseline erythema after 3 months of daily use. Patient-reported outcomes after use of topical brimonidine $0.33 \%$ gel support patients' general acceptability of this treatment option. ${ }^{31}$

Data on efficacy, safety, and patient acceptability of topical brimonidine gel $0.33 \%$ for facial erythema of rosacea are limited to studies with $<1$ year follow-up and case reports. Case reports have been included to highlight several instances of contact dermatitis and rebound erythema in patients who used topical brimonidine gel, in contrast to the relatively low incidence of these adverse events in early studies. Only one study has evaluated the use of topical brimonidine gel with concomitant rosacea or acne medications or in patients with three or more inflammatory lesions. ${ }^{32}$ Although the current data establishes brimonidine topical gel as a safe and effective treatment for erythema associated with rosacea, further studies should aim to examine its long-term efficacy and safety in combination with other rosacea treatments. ${ }^{24}$ 


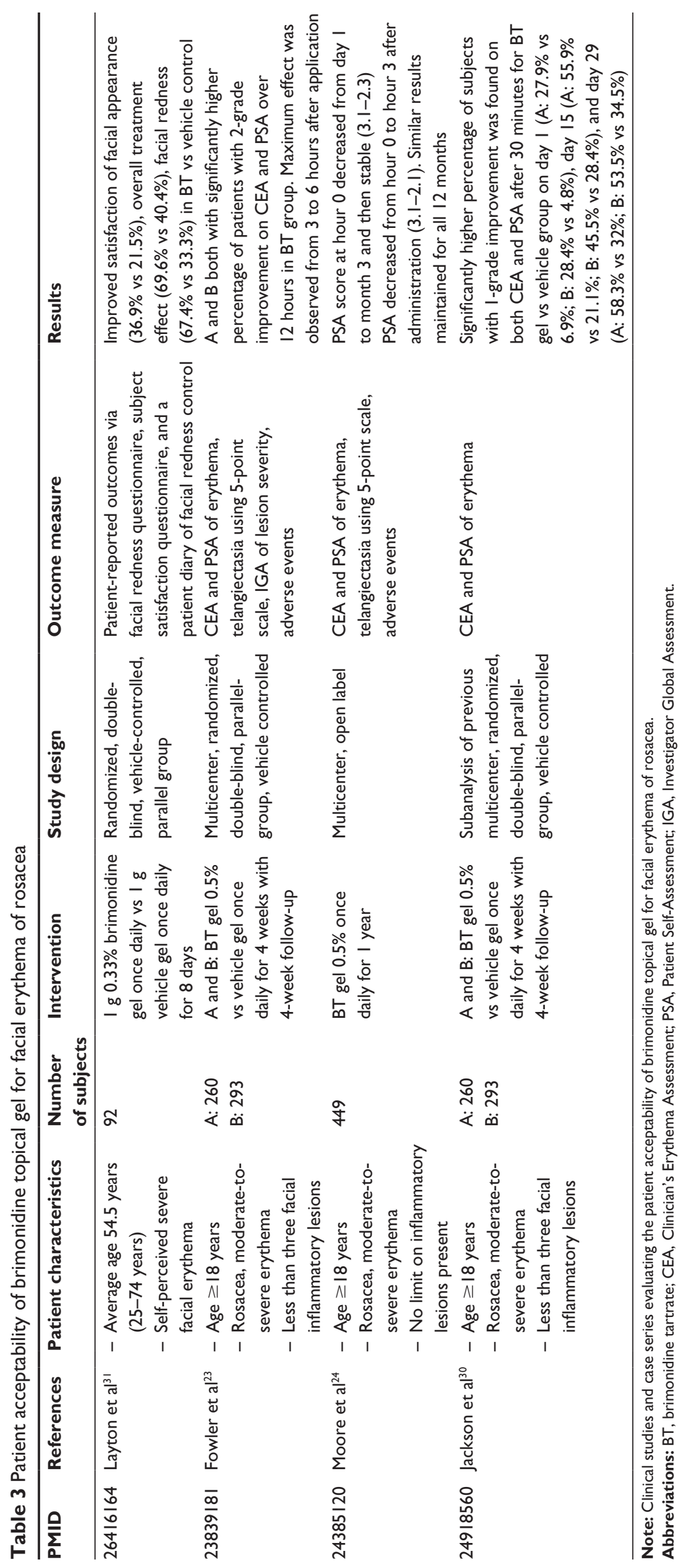


Rosacea treatment presents a unique challenge. Brimonidine topical gel $0.33 \%$ is a novel treatment agent for persistent erythema of rosacea. Though monotherapy may be beneficial in patients with ETR, combination therapy with other rosacea and acne medications may be necessary in patients with features of both ETR and PPR. The shortterm and long-term erythema control offered by brimonidine tartrate gel has its advantages. Patients seeking short-term erythema control for specific events may benefit from asneeded use, whereas those seeking long-term erythema control may opt for daily use resulting in reduced baseline erythema. Controlling erythema in rosacea is a difficult task, but brimonidine topical gel $0.33 \%$ has proven to be a useful addition to the current treatment arsenal.

\section{Disclosure}

Dr Steven R Feldman has received research, speaking, and/or consulting support from a variety of companies, including Galderma, GSK/Stiefel, Almirall, Leo Pharma, Baxter, Boeringer Ingelheim, Mylan, Celgene, Pfizer, Valeant, Taro, Abbvie, Cosmederm, Anacor, Astellas, Janssen, Lilly, Merck, Merz, Novartis, Regeneron, Novan, Parion, Qurient, National Biological Corporation, Caremark, Advance Medical, Sun Pharma, Suncare Research, Informa, UpToDate, and National Psoriasis Foundation. He also consults for others through Guidepoint Global, Gerson Lehrman, and other consulting organizations. He is the founder and majority owner of DrScore. He is the founder and part owner of Causa Research, a company dedicated to enhancing patients' adherence to treatment. The authors report no other conflicts of interest in this work.

\section{References}

1. Tan J, Blume-Peytavi U, Ortonne JP, et al. An observational crosssectional survey of rosacea: clinical associations and progression between subtypes. Br J Dermatol. 2013;169(3):555-562.

2. Webster GF. Rosacea. Med Clin North Am. 2009;93(6):1183-1194.

3. Powell FC. Clinical practice. Rosacea. N Engl J Med. 2005;352(8): 793-803.

4. Wilkin J, Dahl M, Detmar M, et al. Standard classification of rosacea: report of the National Rosacea Society Expert Committee on the classification and staging of rosacea. J Am Acad Dermatol. 2002; 46(4):584-587.

5. van Zuuren EJ, Kramer S, Carter B, Graber MA, Fedorowicz Z. Interventions for rosacea. Cochrane Database Syst Rev. 2011;(3):CD003262. doi:10.1002/14651858.CD003262.pub4.

6. Abram K, Silm H, Maaroos H-I, Oona M. Risk factors associated with rosacea. J Eur Acad Dermatol Venereol. 2010;24(5):565-571.

7. McAleer MA, Fitzpatrick P, Powell FC. Papulopustular rosacea: prevalence and relationship to photodamage. J Am Acad Dermatol. 2010; 63(1):33-39.

8. Jackson JM, Knuckles M, Minni JP, Johnson SM, Belasco KT. The role of brimonidine tartrate gel in the treatment of rosacea. Clin Cosmet Investig Dermatol. 2015;8:529-538.
9. Yamasaki K, Gallo RL. The molecular pathology of rosacea. J Dermatol Sci. 2009;55(2):77-81.

10. Yamasaki K, Di Nardo A, Bardan A, et al. Increased serine protease activity and cathelicidin promotes skin inflammation in rosacea. Nat Med. 2007;13(8):975-980.

11. Zhao YE, Wu LP, Peng Y, Cheng H. Retrospective analysis of the association between Demodex infestation and rosacea. Arch Dermatol. 2010;146(8):896-902.

12. Lacey N, Delaney S, Kavanagh K, Powell FC. Mite-related bacterial antigens stimulate inflammatory cells in rosacea. Br J Dermatol. 2007;157(3):474-481.

13. Brauchle M, Funk JO, Kind P, Werner S. Ultraviolet B and $\mathrm{H} 2 \mathrm{O} 2$ are potent inducers of vascular endothelial growth factor expression in cultured keratinocytes. J Biol Chem. 1996;271(36): 21793-21797.

14. Bielenberg DR, Bucana CD, Sanchez R, Donawho CK, Kripke ML, Fidler IJ. Molecular regulation of UVB-induced cutaneous angiogenesis. J Invest Dermatol. 1998;111(5):864-872.

15. Longuet-Perret I, Schmitt D, Viac J. Tumour necrosis factor-alpha is involved in the contrasting effects of ultraviolet B and ultraviolet A1 radiation on the release by normal human keratinocytes of vascular permeability factor. Br J Dermatol. 1998;138(2):221-224.

16. Sulk M, Seeliger S, Aubert J, et al. Distribution and expression of non-neuronal transient receptor potential (TRPV) ion channels in rosacea. J Invest Dermatol. 2012;132(4):1253-1262.

17. Cribier B. Rosacea under the microscope: characteristic histological findings. J Eur Acad Dermatol Venereol. 2013;27(11):1336-1343.

18. Steinhoff M, Buddenkotte J, Aubert J, et al. Clinical, cellular, and molecular aspects in the pathophysiology of rosacea. J Investig Dermatol Symp Proc. 2011;15(1):2-11.

19. Aubdool AA, Brain SD. Neurovascular aspects of skin neurogenic inflammation. J Investig Dermatol Symp Proc. 2011;15(1):33-39.

20. Del Rosso JQ. Advances in understanding and managing rosacea. Part 1: connecting the dots between pathophysiological mechanisms and common clinical features of rosacea with emphasis on vascular changes and facial erythema. J Clin Aesthet Dermatol. 2012;5(3): 16-25.

21. emc [webpage on the Internet]. Mirvaso $3 \mathrm{mg} / \mathrm{g}$ Gel - summary of product characteristics (SPC) - (eMC). Available from: http:// www.medicines.org.uk/emc/medicine/28682/SPC/Mirvaso+3 $\mathrm{mg}+\mathrm{g}+\mathrm{Gel} /$ \#PHARMACOLOGICAL_PROPS. Accessed January 29, 2017.

22. Fowler J, Jarratt M, Moore A, et al. Once-daily topical brimonidine tartrate gel $0.5 \%$ is a novel treatment for moderate to severe facial erythema of rosacea: results of two multicentre, randomized and vehiclecontrolled studies. Br J Dermatol. 2012;166(3):633-641.

23. Fowler JJ, Jackson M, Moore A, et al. Efficacy and safety of oncedaily topical brimonidine tartrate gel $0.5 \%$ for the treatment of moderate to severe facial erythema of rosacea: results of two randomized, double-blind, and vehicle-controlled pivotal studies. J Drugs Dermatol. 2013;12(6):650-656.

24. Moore A, Kempers S, Murakawa G, et al. Long-term safety and efficacy of once-daily topical brimonidine tartrate gel $0.5 \%$ for the treatment of moderate to severe facial erythema of rosacea: results of a 1-year open-label study. J Drugs Dermatol. 2014;13(1):56-61.

25. Lowe E, Lim S. Paradoxical erythema reaction of long-term topical brimonidine gel for the treatment of facial erythema of rosacea. J Drugs Dermatol. 2016;15(6):763-765.

26. Cookson H, McFadden J, White J, White IR. Allergic contact dermatitis caused by Mirvaso(R), brimonidine tartrate gel $0.33 \%$, a new topical treatment for rosaceal erythema. Contact Dermatitis. 2015;73(6): 366-367.

27. Ilkovitch D, Pomerantz RG. Brimonidine effective but may lead to significant rebound erythema. J Am Acad Dermatol. 2014;70(5): e109-e110.

28. Routt ET, Levitt JO. Rebound erythema and burning sensation from a new topical brimonidine tartrate gel 0.33\%. J Am Acad Dermatol. 2014;70(2):e37-e38. 
29. Swanson LA, Warshaw EM. Allergic contact dermatitis to topical brimonidine tartrate gel $0.33 \%$ for treatment of rosacea. J Am Acad Dermatol. 2014;71(4):832-833.

30. Jackson JM, Fowler J, Moore A, et al. Improvement in facial erythema within 30 minutes of initial application of brimonidine tartrate in patients with rosacea. J Drugs Dermatol. 2014;13(6): 699-704.
31. Layton AM, Schaller M, Homey B, et al. Brimonidine gel $0.33 \%$ rapidly improves patient-reported outcomes by controlling facial erythema of rosacea: a randomized, double-blind, vehiclecontrolled study. J Eur Acad Dermatol Venereol. 2015;29(12): $2405-2410$

\section{Publish your work in this journal}

Patient Preference and Adherence is an international, peer-reviewed, open access journal that focuses on the growing importance of patient preference and adherence throughout the therapeutic continuum. Patient satisfaction, acceptability, quality of life, compliance, persistence and their role in developing new therapeutic modalities and compounds to optimize clinical outcomes for existing disease states are major areas of interest for the journal. This journal has been accepted for indexing on PubMed Central. The manuscript management system is completely online and includes a very quick and fair peer-review system, which is all easy to use. Visit http://www dovepress.com/testimonials.php to read real quotes from published authors.

Submit your manuscript here: http://www.dovepress.com/patient-preference-and-adherence-journal 